\title{
PORRESPONDENCE
}

\section{Oral Interventions for Obstructive Sleep Apnea}

An Umbrella Review of the Effectiveness of Intraoral Appliances, Maxillary Expansion, and Maxillomandibular Advancement

by Dr. med. dent. Vasiliki Koretsi, Prof. Dr. Theodore Eliades, and Dr. med. dent. Spyridon N. Papageorgiou in issue 12/2018

\section{False Comparison, and Interdisciplinary Approach Is Lacking}

Koretsi and colleagues compared intraoral appliances (IOA, the umbrella term for the abbreviation UKPS [Unterkieferprotrusionsschiene], which is commonly used in Germany) versus placebo and versus no treatment with regard to effectiveness in terms of (1):

- Apnea-hypopnea index (AHI) (up to $5=$ normal, up to $15=\mathrm{ac}-$ ceptable),

- score achieved in the Epworth Sleepiness Scale (ESS) questionnaire, and

- minimum oxygen saturation during sleep.

This comparison is almost meaningless. What should be compared instead is IOA/UKPS versus continuous positive airway pressure (CPAP) as standard treatment. This comparison is completely lacking in the studies compared. Furthermore, lowering the AHI from 60 to 30, for example, is pointless as the affected person continues to experience obstructive sleep apnea syndrome (OSAS), with the described risks for blood pressure, stroke, and reduced life expectancy.

An astonishing key message is alleged on page 2-6: "There is no robust scientific evidence to support treatment of OSA patients with [...] surgical maxillomandibular advancement (MMA)."

Vicini et al. published a prospective randomized controlled study in 2010 of bilateral maxillomandibular advancement versus CPAP treatment. Their study found that both treatments were equivalent with regard to AHI and ESS (2). Such a study for OA/ UKPS in comparison to the gold standard CPAP is lacking in the authors' "umbrella" study. Koretsi et al. are dental surgeons specializing in orthodontics, and with their article title "Oral Interventions for Obstructive Sleep Apnea" they lay claim to an "umbrella study," a meta-analysis of meta-analyses. But is uvulopalatoplasty (UPPP) in its variations, and are other interventions - such as electronically guided hypoglossal nerve stimulators ("tongue pacemakers") — not therapeutic oral interventions? Meta-analyses in sleep medicine should be conducted in an interdisciplinary manner, by involving other medical disciplines such as abdominal surgery, otorhinolaryngology, cardiology, oromaxillofacial surgery, neurology and pulmonology.

DOI: 10.3238/arztebl.2018.0556a

\section{References}

1. Koretsi V, Eliades T, Papageorgiou SN: Oral interventions for obstructive sleep apnea-an umbrella review of the effectiveness of intraoral appliances, maxillary expansion, and maxillomandibular advancement. Dtsch Arztebl Int 2018; 115: 200-7.

2. Vicini C, Dallan I, Campanini A, et al.: Surgery vs ventilation in adult severe obstructive sleep apnea syndrome. Am J Otolaryngol 2010; 31: 14-20.

\section{In Reply:}

We thank our readers and correspondents for their interest in our umbrella review of therapeutic oral interventions in obstructive sleep apnea (OSA) (1).

As we stated in our materials and methods section, the objective and protocol of our study were defined a priori. The study objective was to assess the evidence base underlying oral interventions in the treatment of OSA, which are of interest to, but not necessarily restricted to dentists or orthodontists. For this reason, continuous positive airway pressure (CPAP), uvulopalatoplasty, and electronically guided hypoglossal nerve stimulators did not fall under this objective. The study by Vicini et al. (2) was excluded from our review since it compared maxillomandibular advancement (MMA) with CPAP, which was not consistent with our selection criteria. Nonetheless, we acknowledge CPAP as first-line therapy for OSA and mentioned this in our discussion section, where the study by Vicini et al. (2) is cited, among others.

We wish to point out that the original objective in our study protocol was to consider not only randomized but also nonrandomized prospective studies. Unfortunately, such studies with untreated or placebo-matched control patients for MMA and maxillary expansion could not be identified. We consider this fact astonishing because these procedures are an integral part of the standard protocols for OSA in many hospitals. Therefore, it was concluded that there is no robust scientific evidence to support these treatments at the present time.

Finally, we agree that interdisciplinary approaches have an important role in clinical decision-making, as long as they are supported by high quality studies.

DOI: 10.3238/arztebl.2018.0556b

\section{References}

1. Koretsi V, Eliades T, Papageorgiou SN: Oral interventions for obstructive sleep apnea-an umbrella review of the effectiveness of intraoral appliances, maxillary expansion, and maxillomandibular advancement. Dtsch Arztebl Int 2018; 115: 200-7.

2. Vicini $C$, Dallan I, Campanini $A$, et al.: Surgery vs ventilation in adult severe obstructive sleep apnea syndrome. Am J Otolaryngol 2010; 31: 14-20.

Dr. med. dent. Vasiliki Koretsi

Abteilung für Kieferorthopädie, Universitätsklinikum Regensburg koretsi.vasiliki@gmail.com

\section{Conflict of interest statement}

The authors of both contributions declare that no conflict of interest exists. 